\title{
Las metáforas en la cultura escolar de una escuela del nivel inicial.
}

\section{Metaphors in the school culture of an initial level school.}

Carrasco Pérez, David Alberto

Pontificia Universidad Católica del Perú

a20194193@ @ucp.edu.pe

https://orcid.org/0000-0003-0698-3928

\author{
Sánchez Huarcaya, Alex Oswaldo \\ Pontificia Universidad Católica del Perú \\ aosanchezh@pucp.edu.pe \\ https://orcid.org/0000-0003-3902-5902
}

Resumen: En la cultura escolar, las metáforas son una forma de comprender la dinámica de la escuela, por lo cual, los sujetos construyen ciertas expresiones que son compartidas y transmitidas. Este estudio tiene como finalidad responder al siguiente problema: ¿cuáles son las metáforas utilizadas por los docentes y director de una escuela del nivel inicial? Por ello, se establece como objetivo analizar las metáforas utilizadas por los docentes y director de una escuela del nivel inicial ubicada en San Martín de Porres. Para el recojo de información se entrevistó a los docentes y director de la institución elegida. Como conclusiones, se identificaron cuatro orientaciones de las metáforas: Los valores están en crisis, cada niño es diferente, sentido de pertenencia, $\mathrm{y}$ formas diferentes de pensar.

Palabras clave: Metáforas, Cultura Escolar, Gestión, Valores, Costumbres.

\begin{abstract}
In school culture, metaphors are a way of understanding the dynamics of the school, therefore, the subjects construct certain expressions that are shared and transmitted. This study has as purpose to answer the following problem iwhat are the metaphors used by teachers and principal of an entry level school? Thus, set as goal to analyze metaphors used by teachers and principal of an entry level school located in San Martín de Porres. To pick up information, teachers and principal of the chosen institution were interviewed. As conclusions, there were identified four orientations of metaphors. Values are in crisis, each child is different, sense of belonging, and different ways of thinking.
\end{abstract}

Keywords: Metaphors, School Culture, Management, Values, Customs.

\section{COMPRENDIENDO LAS METÁFORAS RELACIONADAS A LA GESTIÓN ESCOLAR}

Actualmente, la cultura escolar es uno de los pilares para mantener una institución educativa en el tiempo y esto tiene gran influencia en las partes involucradas, tales como las instituciones gubernamentales, los directivos, profesores, administrativos, alumnos y padres de familia. Por ejemplo, Airasca (2012) "señala que los seres

Las metáforas en la cultura escolar de una escuela del nivel inicial - Carrasco, David \& Sánchez, Alex.

DOI: https://doi.org/10.18800/cige2020.009 
humanos, nos movemos y decidimos en gran medida en función de las creencias. Además, indica que, los procesos de toma de decisiones y la conducta de los docentes están directamente influidos por la forma como el docente concibe su propio mundo profesional" (p. 55). Es por ello que a lo largo de este texto queremos que se comprendan las metáforas dentro de una organización educativa.

Para esto, hablaremos de su significado, clasificación y sus características; asimismo, enfatizaremos también en su importancia, tomando en cuenta el impacto positivo y negativo que llegan a tener en el sistema educativo.

Para comprender cómo influyen las metáforas dentro de una organización educativa, primero debemos conocer el significado de metáfora, y para ello nos apoyamos en la definición de Balci (2011), quien la considera como una explicación sobre lo que sucede en la escuela y es expresada a manera de analogía. En ese sentido, podemos recalcar que una metáfora comprende la creencia de una persona sobre un objeto, sujeto o tema específico, tomando en cuenta la experiencia que obtenida anteriormente y relacionándola con una palabra ya conocida en su lenguaje.

Considerando esto, en las escuelas podemos escuchar muchos significados por parte de los docentes, así como de los estudiantes, directivos o padres de familia. Por ejemplo, en una institución educativa en donde, debido a la cantidad del alumnado, se debe aperturar un aula más para el mismo grado, los tutores están en constante comparación por parte de los padres de familia o hasta de sus mismos colegas, ya que se puede enfatizar mucho en la relación experiencia - edad del profesor, "ese profesor no sabe tratar a los alumnos porque ya es mayor de edad"; "al profesor novel aún le falta experiencia para enseñar, no sabe dirigir un aula."

Con esto, deseamos se entienda el por qué es necesario trabajar el tema de las metáforas en la educación, considerando que comprenderlas nos permitirá conocer más a la organización desde sus propios actores o personas vinculadas a ella. Para esto, se profundizará en la clasificación de las metáforas educativas.

Primero, en cuanto a las metáforas educativas hacia los directivos, estas son especialmente inducidas por cómo es el trato hacia los alumnos, padres de familia, profesores y coordinadores, y de qué manera están cumpliendo los objetivos de la institución. El director siempre será el centro de atención en la escuela, desde que ingresa hasta que se retira, es por ello que un director novel deberá apoyarse constantemente en los profesores más experimentados, llevando en su gestión la colaboración organizacional, tal y como cita Gonzales $(2015$, p. 27) "la metáfora de la inteligencia colaborativa resulta especialmente fértil para dar cuenta del modo en que los individuos dentro una organización son capaces de coordinar acciones en pos de alcanzar sus objetivos".

Segundo, metáforas educativas sobre el profesorado. Este tipo de creencias se ha ido arraigando cada vez más al campo de educativo de los docentes, debido a que son, en la mayoría de los casos, el centro de opinión por parte de los alumnos, sean estas críticas constructivas o destructivas. Cerit (2006) señala que los estudiantes describen a los docentes como fuentes de información, amigo, padre; y en otros casos, son considerados como recaudador y desarrollador (Ekiz y Koçyiğit, 2012). Dicho de otro modo, un profesor que ha sabido llegar a sus alumnos será visto no solo como el

Las metáforas en la cultura escolar de una escuela del nivel inicial - Carrasco, David \& Sánchez, Alex.

DOI: https://doi.org/10.18800/cige2020.009 
encargado de impartir conocimiento, sino también se le añadirán títulos honoríficos a su cargo.

Tercero, metáforas educativas sobre el alumnado. Los escolares de hoy llevan consigo una cruz al ser comparados siempre con antiguas generaciones. Comparaciones que se dan por la forma en que pueden adquirir información (búsqueda online) o, en otros casos, por el comportamiento que pueden tener frente a una determinada situación. Es decir, nuestros alumnos están siendo discriminados por factores que no deberían de enfatizar en su persona, sin considerar que aún se encuentran en una etapa de desarrollo educativo. Considerando ello, Inbar (1996) "encontró en su investigación que los estudiantes evaluados crearon metáforas sobre la escuela como fabrica, prisión o zoológico" (p. 80). Entonces, las generaciones de estudiantes no deben ser comparadas, ya que el clima estudiantil al que están expuestos siempre se encontrará en constante cambio.

Por otra parte, podemos encontrar que las metáforas también tienen un impacto negativo en el sistema educativo, ya que se puede tergiversar información en caso se le haya implantado una creencia fuerte a un subsistema o sujeto, creando en cualquiera de estos, características que puedan describirlo de manera incorrecta. Por ejemplo, Montoya (2010) "indica que las metáforas son una parte integrante del lenguaje y del pensamiento y aparecen en las conversaciones cotidianas, aun sin que en ellas tenidas conscientemente en cuenta" (p.76).

En conclusión, las metáforas son fundamentales en las actividades humanas porque dan cuenta de su cultura, desde sus tensiones, beneficios o realidad misma, así como de ideas nuevas (Molina, 2001; Valero-García y Navarro, 2008). De esta manera, uno comprende lo que se vive en la escuela, desde sus relaciones, interacciones, modos de actuar y vivencias del día a día. Por lo tanto, "las metáforas pueden servir como instrumentos de análisis de las prácticas y experiencias" (Oxford et al., 1998, citado en Alarcón, Díaz, Tagle, Ramos y Quintana, 2014b, p.29) y vividas en la escuela.

\section{DISEÑO METODOLÓGICO}

La investigación responde a la pregunta: ¿cuáles son las metáforas utilizadas por los docentes y director de una escuela del nivel inicial? Por ello, se establece como objetivo analizar las metáforas utilizadas por los docentes y director de una escuela del nivel inicial de una institución educativa ubicada en San Martin de Porres.

La investigación es cualitativa, porque busca informar con objetividad, claridad y precisión acerca del mundo social de los docentes, considerando que son capaces de aprender e interpretar el mundo, así como de las experiencias de los demás (Monje, 2011; Herrera, Miranda, Pérez \& Koch, 2008). En este caso, serán las metáforas de las docentes y director en relación a la gestión escolar y aprendizaje, las cuales "emergen desde las interpretaciones" (Alarcón, Díaz, Tagle, Ramos y Quintana, 2014a, p.6) de los mismos docentes y director, que se transmiten día a día, como parte de la cultura escolar. Para este tipo de investigación consideramos la técnica de la entrevista, porque nos permitió recoger los pareceres y lo que experimentan día a día en la escuela (Lodico, Spaulding y Voegtle, 2006)

Las metáforas en la cultura escolar de una escuela del nivel inicial - Carrasco, David \& Sánchez, Alex.

DOI: https://doi.org/10.18800/cige2020.009 
En relación al instrumento de recojo de información denominado guía de preguntas, se validó con un especialista, quien se encargó de verificar su coherencia y contenido, además de su relación con el objetivo de estudio, así como su pertenencia. Luego, se procedió a su aplicación y al recojo de información. El instrumento se aplicó a tres profesoras del nivel inicial (aulas de tres, cuatro y cinco años) y al director de la escuela privada ubicada en el distrito de San Martín de Porres, perteneciente a la Ugel 02. Las entrevistas fueron realizadas de manera presencial en la oficina de coordinación del nivel inicial de la institución durante un mes.

En relación a las entrevistadas, la primera profesora se encuentra actualmente a cargo del aula de tres años del nivel inicial, y tiene más de cinco años laborando en la institución. A esta docente se le asignó el código "DO1", el cual significa docente $\mathrm{n}^{\circ}$ 1. La segunda profesora se encuentra a cargo del aula de cuatro años del nivel inicial, y tiene más de diez años laborando en el colegio, con código "DO2". La tercera profesora del nivel inicial, tiene a cargo el aula de cinco años, y se le asignó el código "DO3". Estos códigos serán utilizados en el análisis e interpretación de resultados.

Asimismo, se entrevistó al director de la escuela, el cual se encuentra dirigiendo la institución por más de cinco años, asignándosele el código "DI".

Luego de recoger la información, se procedió a su transcripción, para lo que se elaboró una matriz de hallazgos. Esta matriz consideró tres secciones (columnas) para ubicar la respuesta, hallazgo y elemento emergente. Luego de ubicar las respuestas en la columna respectiva, se procedió a su análisis para identificar los hallazgos y, por último, para interpretarla se utilizó, manualmente, la técnica de codificación abierta u open coding, la cual tiene como objetivo descomponer, examinar y comparar la información obtenida (Kvale, 2011). En nuestra matriz se le denominó elemento emergente, porque a partir del análisis se reducía cada hallazgo a su mínima expresión, es decir, una frase de pocas palabras. Luego, se buscaron los que eran similares y, de esa manera, se obtuvieron las metáforas, las cuales fueron agrupadas. En la tabla 1 las denominamos como orientación de la metáfora.

Finalmente, a partir de la orientación de las metáforas se organizó la interpretación de hallazgos y se consideró la literatura revisada. Previo a esto, debemos confirmar que respetamos el principio ético de la investigación, integridad científica, por lo que a todos los entrevistados se les presentó el consentimiento informado, donde tomaban conocimiento de su participación en el estudio y que lo expresado en las entrevistas serviría solamente para este fin.

\section{INTERPRETACIÓN DE HALLAZGOS}

La interpretación de los hallazgos consistió en el análisis de las respuestas que dieron las docentes del nivel inicial y el director de la institución. Es relevante considerar que la interpretación de las respuestas sirve de referencia para encaminar el análisis de los hallazgos encontrados.

A continuación, presentamos las metáforas identificadas por los entrevistados a partir de sus respuestas, organizadas según su significado.

Las metáforas en la cultura escolar de una escuela del nivel inicial - Carrasco, David \& Sánchez, Alex.

DOI: https://doi.org/10.18800/cige2020.009 
Congreso Internacional de Gestión Educativa, 06 y 07 de noviembre de 2020.

Pontificia Universidad Católica del Perú

\subsection{Los valores están en crisis}

Analizando las respuestas de acuerdo a los valores de la escuela, se percibe que el personal entrevistado considera que la institución está enfocada en la formación integral de los alumnos, pero no recibe el apoyo necesario en sus hogares, por lo que existe un "abismo total de los valores."

"De hecho se ha perdido un montón porque con este código del niño... los estudiantes aprovechan de esa situación y estamos sujetos a equivocarnos y por eso que no se está cumpliendo como debería ser no entonces hay un abismo total de los valores, que, si comparamos tiempos pasados al actual no, los valores que tienen los chicos de la actualidad difiere mucho a los valores antiguos." (DI).

La metáfora enunciada responde al problema de valores actual en las familias, en relación a lo que se brinda en los hogares, siendo este segundo espacio de poca ayuda o de no reafirmación de lo que se trabaja en la escuela. Aunque esto difiere por lo expresado por el DI, "los valores que tienen los chicos de la actualidad difieren mucho de los valores antiguos", es decir, no es que estos no existan, sino que los valores de ahora son diferentes a los considerados en la antigüedad, posiblemente los tomados en cuenta por los padres. Pero se debe resaltar que, si bien hay una mirada de poca atención a los valores tradicionales, también los consideran importantes, por lo que son reforzados por las docentes. Ante esto podemos leer "Esos valores son muy importantes para mí, que como mama, como madre de familia y como docente son importantes porque yo a mis chicos les inculco desde chiquititos los valores, y ellos saben los valores" (D03).

\subsection{Cada niño es diferente}

Lo expresado se puede visualizar a partir de la siguiente metáfora " $L a$ consciencia del alumno es dificil de medir" (DI), la cual nos da a entender que cada estudiante es diferente y es difícil conocer lo que piensan, creen o, incluso, imaginan, aunque eso se puede visualizar "en las acciones. Acá falta bastante compromiso de los padres y de los demás agentes actuantes en su entorno, en su contexto del alumno, como institución hacemos nosotros lo posible" (DI).

Lo explicado hasta el momento puede vincularse con la siguiente metáfora "cada niño es un mundo", y esto puede estar vinculado con lo comentado anteriormente, de que a cada niño se le puede conocer a través de sus expresiones y acciones, de ahí que sabemos sobre sus necesidades, inquietudes, intereses y diversas características propias de su ser, aunque también son influenciados por otras personas y lo que sucede en la comunidad. Esto lo podemos complementar en el siguiente hallazgo "Cada niño es un mundo, como hoy siempre digo, como tú no hay nadie, querrán imitarnos, pero no jamás van a poder comparar, porque uno es especial no y poder trabajar con cada carácter, con cada niñito..." (DO2).

Las metáforas en la cultura escolar de una escuela del nivel inicial - Carrasco, David \& Sánchez, Alex.

DOI: https://doi.org/10.18800/cige2020.009 
Congreso Internacional de Gestión Educativa, 06 y 07 de noviembre de 2020.

Pontificia Universidad Católica del Perú

Tenemos una discusión constante sobre qué conciben los padres por educación y por qué eligen una escuela, e incluso, algunos no se cuestionan por la elección de la escuela para sus hijos, de ahí que emerge la siguiente metáfora "pintar un mundo que no es."

Esta expresión la podemos ampliar leyendo el siguiente hallazgo: "Pintarle un mundo que no es, no, como hace mucho tiempo una mama decía, yo solamente la pongo acá porque quiero que esté en un colegio particular, a la señora no le interesaba si su hija iba a aprender o no iba a aprender, entonces vive en una burbuja, en un mundo de fantasía..." (DO1).

Esa metáfora responde el interés que tiene el padre de ubicar al hijo en un colegio particular sin conocer, posiblemente, la propuesta pedagógica y lo peor aún es que no cuenta con la capacidad económica para costearlo. En una sociedad como la nuestra, en muchos casos, los padres reducen su concepción de educación por el de status, es decir, matriculan a sus hijos en centros privados para diferenciarlos de los estudiantes que van a escuelas públicas.

A continuación, presentamos la siguiente metáfora "incluirse para poder salir al mundo" (D03), que expresa la posibilidad de que la institución educativa forme de la mejor manera, sin diferencias, es decir, incluyendo a todos, aunque una de las docentes expresa las dificultades de los estudiantes al ingresar a la escuela "Le costó adaptarse, le costó adaptarse a los niños" (D03).

Ese proceso de incluir a un estudiante en la institución educativa, de incorporarlo a una escuela regular, es planteado por una docente a través de la siguiente metáfora "yo no la puedo tener en una burbuja" (D03), es decir, debe ser parte de un entorno abierto porque la realidad es así. Cuando salga a la calle conocerá todo tipo de personas, por eso es que expresa "ningún niño con cierta discapacidad puede estar en una burbuja, incluirse para poder salir al mundo, incluir, incluirlo a la sociedad, prepararlo en el colegio para incluirlo en la sociedad, eso es lo que se trata de hacer." (DO3).

Esta metáfora responde a las posibilidades que se le brinda al estudiante incluido para salir adelante, es decir, los profesores tienen claro que la preparación que dan a los estudiantes inclusivos les dará la posibilidad de progresar y ser insertados en la sociedad. El ser aceptado, integrado y formado en una escuela regular, tendrá una repercusión positiva posteriormente, de ahí que complementamos la idea con el siguiente hallazgo "inicial somos los semilleros" (DO2).

\subsection{Sentido de pertenencia}

Lo entendemos como sentirse parte de algo, en este caso, de una institución.

Tenemos la siguiente metáfora "Somos una familia." Esta es una expresión recurrente que se utiliza en las instituciones porque tratan de expresar que se han fortalecido los vínculos como una familia, lo cual podemos leer en el siguiente hallazgo "por tener tres locales no quiere decir que somos ajenos, como te digo, somos una familia, nosotros somos de inicial, pero tenemos compañeras en

Las metáforas en la cultura escolar de una escuela del nivel inicial - Carrasco, David \& Sánchez, Alex.

DOI: https://doi.org/10.18800/cige2020.009 
Congreso Internacional de Gestión Educativa, 06 y 07 de noviembre de 2020.

Pontificia Universidad Católica del Perú

primaria y a veces no hay momento en donde nosotros podamos reunirnos." (DO2).

Otra metáfora identificada es "el colegio es de todos los niños", la cual nos da a entender que todos los niños deben ser considerados y no solo un pequeño grupo, lo que expresa la docente en relación a la participación del estudiante en alguna actividad del colegio, "Que debería ser por cada salón su número artístico de aniversario porque es el aniversario, porque el colegio es de todos los niños, no de cuatro parejas de cada salón no, entonces este eso debería ser." (DO3).

La metáfora "tú le das al cien por ciento", está relacionada con la disposición que se tiene ante el trabajo que, en muchos casos, sobrepasa las horas establecidas, "tú le das al cien por ciento, no te importa cuánto te pagan, no importa que es lo que haces sino quieres que la actividad o la propuesta que tiene el colegio salga de la mejor manera, eso falta" (DI).

Esta última metáfora se puede vincular con "ponerse la camiseta", entendida como estar identificado con la escuela, lo que se refleja al trabajar en las diferentes actividades propuestas, aunque en el siguiente hallazgo menciona "Bueno, sí participan los docentes, participan en las actividades, pero hay algunos pues este que, como se dice, no se ponen la camiseta, entonces y unas son más que otras" (DO3). Aquí se presenta el cuestionamiento de que no todos tienen la camiseta puesta, por lo cual no todos trabajan al mismo ritmo, y se cuestiona la identificación con la escuela.

\subsection{Formas diferentes de pensar}

Una metáfora relacionada con la posibilidad de tener apertura a otras ideas es "tener mente abierta". Un hallazgo refleja lo indicado "Se tiene que innovar, para eso se tiene que tener mente abierta." (DO1). Lo interesante de esta expresión es que resalta la palabra innovación, entendida como la acción de generar nuevas ideas o mejorar lo que se viene haciendo, y una condición para aceptarlo es tener mente abierta. En los últimos tiempos, se solicita a los docentes generar innovaciones en sus propias prácticas, igual que a los directivos de las escuelas, pero también queda claro que ante las innovaciones existe resistencia, porque se asume que generarán más tareas o se da una apatía al realizar nuevas actividades, haciendo necesario el estudio de este tema en particular, por eso manifiestan, "tu trabajas donde tú te sientes a gusto" (DO2).

Otra metáfora es "El mundo es un bumerang”. Entendemos que el bumerang, al ser lanzado, regresa, por lo que la analogía sería que "lo que yo haga me regresará en algún momento", y esto puede ser positivo o negativo, es decir, dependerá de lo realizado. Esta idea la podemos visualizar en el siguiente hallazgo "El mundo es un bumerang, da vueltas, siempre vas a dar lo que tú tienes, si tú tienes en tu corazón amor no, cariño, siempre estas siendo empático, eso es lo que tú vas a dar, eso es lo que tú vas a recibir" (DO1).

Las metáforas en la cultura escolar de una escuela del nivel inicial - Carrasco, David \& Sánchez, Alex.

DOI: https://doi.org/10.18800/cige2020.009 
Congreso Internacional de Gestión Educativa, 06 y 07 de noviembre de 2020.

Pontificia Universidad Católica del Perú

Como lo expresa este último hallazgo y ubicándolo en el contexto educativo, se debe considerar el trato hacia los estudiantes o entre colegas, porque como trates te tratarán, de ahí que se debe considerar la empatía, el afecto y el respeto.

\section{CONCLUSIONES}

A través de las metáforas analizadas, hemos podido comprender lo que se vive en la escuela, desde la formación y gestión, así como los modelos mentales de los docentes y director. Ante esto, las organizamos en cuatro orientaciones: Los valores están en crisis, cada niño es diferente, sentido de pertenencia y formas diferentes de pensar.

Las metáforas identificadas nos han llevado a comprender la cultura escolar, desde sus interrelaciones, identificación, formas de pensar y cómo ven al niño. Esto nos invita a repensar el rol del líder y lo que se espera en la formación de los niños, así como la labor de los docentes.

A partir del estudio, se pueden seguir trabajando las metáforas en la escuela y sumar a otros agentes, de esta manera se podría comprender la cultura de la misma y orientar ciertas acciones que potencien y fortalezcan la propuesta de esta en favor de los estudiantes y docentes.

\section{Agradecimientos}

A mis padres, Alberto y Sara, por haberme apoyado y aconsejado cuando más lo necesite. A mi asesor, Alex, por brindarme el conocimiento necesario para construir las bases del camino de la investigación. Y a las docentes y director de la escuela que formaron parte de esta investigación.

\section{Referencias bibliográficas}

Airasca, D. (2012). La cultura escolar ¿Una aproximación a la cultura sedentaria? Buenos Aires, Argentina: Teseo.

Alarcón, P. Díaz, C., Tagle, T., Ramos, L. y Quintana, M. (2014a). Metáforas para profesor y estudiante de pedagogía, en un grupo de estudiantes de pedagogía chilenos. Revista Actualidades Investigativas en Educación, 14(2), 1-31. Recuperado de https://www.scielo.sa.cr/pdf/aie/v14n2/a17v14n2.pdf

Alarcón, P. Díaz, C., Tagle, T., Ramos, L. y Quintana, M. (2014b). Conceptualizaciones metafóricas sobre el rol del profesor en estudiantes de pedagogía. Estudios

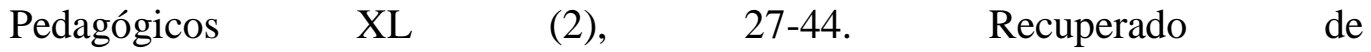
https://scielo.conicyt.cl/pdf/estped/v40n2/art02.pdf

Balci, A. (2011). Turkish teachers' and supervisors' metaphorical perceptions about supervisors. International Research Journals, 2(10), 1602-1610.

Cerit, Y. (2006). School Metaphors: The views of students, teachers and administrators. Educational Sciences: Theory \& Practice, 6(3), 692-699.

Las metáforas en la cultura escolar de una escuela del nivel inicial - Carrasco, David \& Sánchez, Alex.

DOI: https://doi.org/10.18800/cige2020.009 
Congreso Internacional de Gestión Educativa, 06 y 07 de noviembre de 2020.

Pontificia Universidad Católica del Perú

Ekiz, D. \& Koçyiğit, Z. (2013). Exploring primary school teachers' metaphors concerning “teacher”. Kastamonu Education Journal, 21(2), 439-458.

Gonzales, F. (2015). Las metáforas organizacionales en la era de la virtualidad. Revista Argentina de Investigación en Negocios, 19-30. Recuperado de: http://ppct.caicyt.gov.ar/index.php/rain/article/view/v1n1a04

Herrera, M., Miranda, C., Pérez, H. \& Koch, T. (2008). Necesidades de educación permanente de docentes técnicos. Estudios Pedagógicos, 34(1), pp. 7-26. Recuperado de http://www.redalyc.org/articulo.oa?id=173514135001

Inbar, D. E. (1996). The free educational prison. Metaphors and Images. Educational Research, 38(1), 77-92.

Kvale, S. (2011). Las entrevistas en investigación cualitativa. Madrid: Ediciones Morata.

Lodico, M., Spaulding, D. \& Voegtle, K. (2006). Methods in educational research. From theory to practice. San Francisco, USA: Jossey-Bass.

Molina, A. (2001). Las educadoras y sus metáforas educativas: dos investigaciones en acción. Anales de pedagogía, (19), 201-220. Recuperado de https://revistas.um.es/analespedagogia/article/download/284941/206631/

Monje, C. (2011). Metodología de la investigación cuantitativa y cualitativa. Guía didáctica. Neiva: Universidad Surcolombiana.

Montoya Restrepo, L. A., Montoya Restrepo, I. A. \& Castellanos Domínguez, O. F. (2010). La metáfora organizacional: alternativa de entendimiento procedente de otras ciencias. Red de Revistas de América Latina el Caribe, España y Portugal, 8(1), 75-86. Recuperado de: http://www.redalyc.org/pdf/909/90920479004.pdf

Valero-García, M. y Navarro, J. (2008). Diez metáforas para entender (y explicar) el nuevo modelo docente para el EEES. @tic Revista d'innovació educativa, (1), 113. Recuperado de http://bioinfo.uib.es/ joemiro/semdoc/diezMetaforas.pdf

Las metáforas en la cultura escolar de una escuela del nivel inicial - Carrasco, David \& Sánchez, Alex.

DOI: https://doi.org/10.18800/cige2020.009 